Aburatani, Hiroyuki

[1]

\section{Classification of hepatocellular carcinoma with gene expression profiling analysis}

\author{
Hiroyuki Aburatani, Yasushi Midorikawa, Shuichi Tsutsumi \\ \& Yuko Kobune
}

Genome Science Division, Research Center for Advanced Science and Technology, University of Tokyo, Tokyo, Japan

We have attempted classification of hepatocellular carcinoma using gene expression profiling analysis. To identify the genes associated with liver carcinogenesis and the progression of the carcinoma, we analyzed RNAs from 12 liver tumors and 8 non-tumor tissues using GeneChip HuFL arrays, histologically classifying liver tumors as either well differentiated (WD) or moderately differentiated (MD). The two-way clustering method (Pearson correlation) was not effective in distinguishing among cancerous and noncancerous tissues. We sorted approximately 5,600 genes by their degree of correlation with the histological classes to arrive at a list of 40 genes (predictor genes) of significant predictive value in classifying non-cancerous tissues and WD and MD tumors. We then applied the weighted voting algorithm to each pair among the three groups and successfully predicted the class on two-dimensional scatterplots on the basis of prediction strength. Multistep carcinogenesis in liver can be observed in a single tumor with a nodule-within-nodule appearance, in which the outer tumor is WD and the inner one is MD. The expression levels of 74 genes were elevated over threefold in MD in comparison with WD. We applied the Mann-Whitney U-test to 10 liver cancers (5 WDs and $5 \mathrm{MDs}$ ) and found 12 genes to be significantly elevated in expression levels in MD tumors $(P<0.05)$. Evaluation of an additional 12 tumors by quantitative polymerase chain reaction with reverse transcription validated those 12 genes ( 7 of which were also on the list of predictor genes), implying that they are involved in de-differentiation of liver cancer. Our statistical approach to gene expression profile data was therefore effective in cancer classification and the study of cancer progression.

Adya, Neeraj

\section{Characterizing gene expression profile of Inv(16)-associated leukemia}

N. Adya ${ }^{1,2}$, Y. Chen ${ }^{1}$, E. Hilgenfeld ${ }^{3}$, J. Khan ${ }^{1}$, M. Bittner ${ }^{1}$, H. Serve ${ }^{4}$, W.E. Berdel ${ }^{4}$, P. Meltzer ${ }^{1} \&$ P.P. Liu ${ }^{1}$

${ }^{1}$ National Human Genome Research Institute, National Institutes of Health, Bethesda, Maryland, USA

${ }^{2}$ Abbott Laboratory, USA

${ }^{3}$ National Cancer Institute, National Institutes of Health, Bethesda, Maryland, USA

${ }^{4}$ University of Muenster, Muenster, Germany

Inversion $16(\operatorname{Inv}(16))$ results in a fusion between genes $C B F B$ on the $\mathrm{q}$ arm and MYH11 on the p arm and is associated with acute myeloid leukemia (AML) subtype M4Eo. $C B F B$ encodes a protein $(\mathrm{CBFb})$ that forms a heterodimeric transcription factor with RUNX1/AML1, which itself is disrupted by several chromosome translocations in leukemias. MYH11 encodes the smooth muscle myosin heavy chain (SMMHC). The fusion gene $C B F B-M Y H 11$, through its encoded $\mathrm{CBF} \beta-\mathrm{SMMHC}$ fusion protein, dominant negatively disrupts the normal func- tion of the CBFß/AML1 dimer in gene expression regulation and hematopoiesis. Using cDNA microarray technology we attempted to identify gene expression profiles characteristic to $\operatorname{Inv}(16)$ in comparison with non-Inv(16) AML and normal bone marrow (BM). RNA from five cases of M4Eo AML with Inv(16), seven cases with M4 AML without inv(16) and five normal BM samples was used to generate labeled cDNA probes to hybridize against a $6.7-\mathrm{kD}$ human cDNA microarray. We used a common reference probe generated from the cell line HL60 to co-hybridize with cDNA probes derived from patients, allowing normalization of the expression level and ratio of expression of each gene in each sample relative to HL-60. M4Eo, M4 and normal BM were the principal three clusters or groups of samples observed when the expression ratios were compared using hierarchical clustering and multidimensional scaling (MDS). The maximum difference in gene expression profiles as computed by MDS and assigned as a measure of distance between samples was that for M4Eo and M4 versus normal BM. We have generated a list of weighted genes whose expression differences have the greatest power to distinguish among the groups. Those gene expression changes that are unique to the $\operatorname{Inv}(16)$ cases will be studied further. This study may help us better understand the oncogenic mechanism of $C B F B-M Y H 11$ and provide new therapeutic targets.

Afshari, Cynthia

\section{Combination of functional genomics approaches and microarray analyses to define candidate senescence genes}

Lois Annab ${ }^{1}$, Natalay Kouprina ${ }^{1}$, Vladimir Larionov ${ }^{1}$, Patrick Vojta ${ }^{1}$, Motonobu Katoh ${ }^{2}$, Pierre Bushel ${ }^{1}$, Karla Martin $^{1}$, Mitsuo Oshimura ${ }^{2}$, J. Carl Barrett ${ }^{1} \&$ Cynthia Afshari ${ }^{1}$

${ }^{1}$ National Institute of Environmental Health Sciences, Research Triangle Park, North Carolina, USA

${ }^{2}$ Tottori University, Tottori, Japan

We are using a combination of functional genomics approaches and microarray analysis techniques to identify and clone human senescence genes. One area of focus is identification of a gene in the region of human chromosome 1q42. Past studies of microcell-mediated chromosome transfer experiments revealed senescence gene activity on human chromosome 1 . Subsequent analyses of revertants and radiation-induced selectable tagged fragments of chromosome 1 further narrowed the region of activity to a 5-cM region on $1 \mathrm{q} 42$ that is too large to clone using traditional positional cloning methodologies. In one approach to identifying the gene functionally, transfer of commercially available yeast artificial chromosomes from the $1 \mathrm{q} 42$ region by spheroplast fusion led to identification of a 1.2-Mb artificial chromosome that contains possible senescence activity, as indicated by decreased colony formation of an immortal cell following spheroplast fusion. Analysis of Gene Map 98 revealed 450 defined candidate genes or expressed sequence tags in the 1q42 region. We developed a complementary DNA microarray chip that contains all these candidate genes and expressed sequence tags. We are conducting microarray analysis using a cDNA microarray chip that contains a total of 12,000 genes and expressed sequence tags, including those from the $1 \mathrm{q} 42$ region. We are conducting hybridizations with RNA derived from young, immortal, G1arrested and senescent fibroblast and epithelial cells to identify critical targets of the senescence and immortalization processes. Our goal is to couple the microarray analysis with our physical and functional mapping approaches to identify the candidate gene on 1q42 that is responsible for inducing the senescence phenotype. 